\title{
Construction of Distribution Network Power Supply Reliability Monitoring and Analysis System
}

\author{
Wang Dan ${ }^{1}$, Wu Xiaomei' ${ }^{1,}$ Wang Yuting ${ }^{1}$, Zhu Bo ${ }^{2}$, Wang Jiaying ${ }^{2}$, Meng Yanqing ${ }^{2}$, and \\ Wang Qinghao ${ }^{2}$ \\ ${ }^{1}$ Fuxin Power Supply Company, Liaoning Electric Power Company Limited, State Grid, China \\ ${ }^{2}$ Fushun Power Supply Company, Liaoning Electric Power Company Limited, State Grid, China
}

Keywords: Distribution network power supply reliability, data, monitoring and analysis, frequent power outages, intelligence

\begin{abstract}
In order to make the distribution network construction more digital and intelligent, the company team has built a distribution network power supply reliability monitoring and analysis system by using information technology. This article introduces the "surface-line-point" monitoring and analysis model of this system, Discusses the construction process of the system from data acquisition, data modeling to data analysis, multi-dimensional analysis, intelligent reports, etc. Through the construction of the distribution network power supply reliability monitoring and analysis system, the monitoring and analysis of the distribution network power supply reliability related data is realized, Restore the authenticity and validity of the distribution network data, so as to predict the frequent power outages, reduce the impact of frequent power outages on the reliability of power supply, improve the grid's intelligent operation level, further improve the company's quality service quality, and promote the company's operational efficiency and Double benefits.
\end{abstract}

\section{Introduction}

At present, with the tightening of government supervision and the accelerated advancement of cross-border integration, State Grid Corporation based on serving the party and the overall work of the state, giving full play to the leading role of central enterprises, actively promoting the energy revolution, the digital revolution, and proposing the grandness of building a world-class energy Internet enterprise Objectives. The development of a strong smart grid and the construction of an electric power Internet of Things have combined to form an energy Internet of "three streams in one" of energy flow, business flow, and data flow. Currently, business flow and energy flow have been formed, but data flow is still lacking. Therefore, there is an urgent need to build an enterprise data stream.

In order to deeply implement the national energy security, network power, digital economy and other strategies, promote the digitization, network and intelligent upgrade of enterprises, promote the in-depth integration of energy transformation and information technology, and help the high-quality development of power grids and companies. Due to the problems of weak power grid and low intelligence level, a distribution power supply reliability monitoring and analysis system has been constructed. This system is used to monitor and analyze the power outage of distribution lines, and strive to digitally analyze and improve the reliability of distribution network power supply. The intelligent operation level of the power grid further improves the company's quality service quality and promotes the company's operational efficiency and benefits.

\section{The Original Problem}

In recent years, the company's customer complaints caused by frequent power outages in the distribution network have increased year by year, which has seriously affected the reliability and service quality of the company's power supply. Therefore, monitoring and analysis of the frequent power outages of the distribution network to realize the pre-judgment of frequent power outages has 
become an increase in power supply of the distribution network An important means of reliability.

At present, the company's distribution network does not have terminal equipment for acquisition, and it lacks information technology to automatically obtain information. It can only rely on original management methods such as manual reports for information synthesis. The power outage process and power outage plan in the pmis system also only record a small number of power outages. Information, the full amount of data on power distribution blackouts cannot be obtained, and frequent power outages in the distribution network cannot be accurately predicted and avoided, which restricts the intelligent development of the distribution network. Therefore, the team used the current information technology to build the reliability of the distribution network power supply The monitoring and analysis system realizes the monitoring and analysis of the power supply reliability data of the distribution network, restores the authenticity and validity of the distribution network data, so as to predict the frequent power outages, and make the company's distribution network construction move towards intelligent and digital A powerful step.

\section{Construct Ideas}

\subsection{Establish "Surface-Line-Point" Monitoring and Analysis Model}

From the monitoring method, it mainly uses comparison monitoring and penetration query; from the analysis method, it uses a combination of online and offline data collection methods, and uses "surface-line-point" method to perform detailed data and index data. Mining and analysis to get the overall level and trend of distribution network power supply reliability.

\section{2 "Face" Monitoring Analysis}

The "face" monitoring and analysis is to compare and analyze the detailed data in multiple business systems, find out the differences of the detailed data, and analyze the accuracy and consistency of the data of each business system. Separately, the power outage information and production in the power information collection system The line outage information in the PMIS system and the distribution network repair work order information in the dispatch management system are compared, and the outage time, outage range, and outage nature are analyzed.

\section{3 "Line" Monitoring Analysis}

"Line" monitoring and analysis is to obtain the trend change of the substation detailed data in the electrical energy information collection system, and calculate the daily, monthly and seasonal change trend of the power outage of the line where the substation is located. The trend analysis of the company's power outage information data is used to obtain the overall change Trends, find out the time periods with large changes, and analyze the proportion of impact.

\section{4 "Point" Monitoring Analysis}

"Point" monitoring analysis is to obtain detailed data of power outages in distribution networks, monitor and analyze the causes of each type of power outages, and find out the main factors that cause power outages and low voltages in distribution networks. Perform statistical analysis on the causes of each type of power outages. Important factors.

\section{Build Process}

From the technical implementation point of view, the system is based on real-time detailed data of the marketing electrical energy information collection system, and uses a multi-level synchronous sharing mode to monitor and analyze the power supply situation of the distribution network. The monitoring object is mainly the power outage data of the line, including the length of power outage, the number of power outages, and power outage Nature, power outage distribution, etc., analyze frequent power outages to obtain power outage trends, etc.

First of all, data loading, data calculation, mathematical model, dimensional model are established, and the data source is calculated; secondly, the data is analyzed and processed in the 
manner of data analysis, multi-dimensional analysis, intelligent report, mining analysis, etc. The collected data is analyzed and processed, Screen valuable information; establish a dimensional model of the monitoring object, form a multi-faceted and comprehensive monitoring analysis; establish data formulas and screening conditions for different dimensions in the process, and form data achievements; form a visual, clickable, and highlighting intelligent chart When the construction of distribution network automation has just started, it basically solves the situation in the past that it was impossible to accurately and timely obtain the distribution network outage information. The management department can grasp the distribution of outage events in cities and counties, and analyze the reasons for the outage by dispatching work orders to check.

\section{Technical Characteristics}

Using VBA + MySQL + Apache + Dreamweaver intelligent technology, it can realize the automatic acquisition of the time point data of the station area, calculate the power outage information of the station area, and integrate the function into the browser to automatically calculate the line outage information. The advantages are: 1 . Calculate the power outage information of the line; 2 . The calculation time is short 3. The monitoring process can be automated.

\section{System Workflow}

\subsection{Obtain Marketing Collection Data}

Obtain real-time station voltage data from the marketing electrical energy information collection system, realize real-time monitoring of the operation status of distribution network equipment from the end, and closely connect marketing collection with production and distribution.

\subsubsection{Data Acquisition}

The special and public transformer stations in Fuxin area are basically fully covered and fully collected. The voltage data of the stations automatically obtained by the collection system, combined with other related information (such as line topology information such as the power grid topology map), indirectly generate power outage monitoring for the lines in the stations The required information to realize the trend analysis of power outage lines.

This is a part of the data segment collected from 37 o'clock to 50 o'clock. It can be seen that the red part is the part where the voltage information is missing, and it can be initially judged as the power outage information.

\begin{tabular}{|c|c|c|c|c|c|c|c|c|c|c|c|c|c|}
\hline AK & AL & All & AN & AO & $A P$ & $m$ & AR & & Al & & AV & W & \\
\hline 7-10 & 38 & U39 & U40 & U41-11 & U42 & U43 & U44 & U455-12 & U46 & U47 & U48 & U49-13 & 150 \\
\hline & 233.40 & 232.90 & 233.00 & 232.60 & 233.00 & 232.80 & 231.50 & 232.80 & 233.50 & 234.20 & 233.70 & 233.70 & 35.80 \\
\hline & non & & & & & & & & & 2 & & & \\
\hline & & & & & & & & & & & & & \\
\hline & 103.10 & 103.30 & 103.30 & 103.30 & 103.20 & 103.20 & 103.10 & 103.40 & 103.40 & 103.60 & 103.70 & 03.80 & 104 \\
\hline & 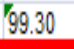 & 6010 & 1000 & 99.70 & 199.60 & 199.50 & 10 & 99.20 & 199.40 & 99.50 & 8.80 & & \\
\hline & & & & 102.40 & & 102. & 0 & 101.80 & & 102.20 & t & 2.70 & \\
\hline & & & & 230.50 & 533 & 1230 & 22 & 232.70 & 230.00 & 230.30 & & 31.80 & 232.50 \\
\hline & 570 & 1.40 & 59 & 226.00 & 230.8 & $n$ & h & 229.60 & 122 & & & 90 & 227.80 \\
\hline & & & & 227 & 231. & & 225 & 230.60 & 226.30 & 90 & 231.30 & 228.20 & 229. \\
\hline & 200 & & & & 11020 & & & & 102.00 & & & 14080 & \\
\hline 000 & 28.10 & & 8 & 22060 & 231.2 & , & 230 & 232.00 & 231.20 & 232.00 & 234. & 233.50 & 3550 \\
\hline & & & & & 230 & & 228 & 227.30 & 230.60 & 0 & 227 & 231.60 & 3120 \\
\hline & & & & & & & & & & & & & \\
\hline & & & & & & & & & & & & & \\
\hline
\end{tabular}

Figure 1 The time of collecting information is low

\subsubsection{Data Processing}

According to the collection cycle, there is voltage at the last time point, there is no voltage at this time point, and this time point is regarded as abnormal; there is no voltage at the last time point, 
there is voltage at this time point, and the last time point is considered to be abnormal. It is regarded as abnormal, and the corresponding abnormal mark is marked. On the same acquisition date and the same station area, as long as the three phases of a, b, and c are abnormal, all the records of the three phases of abc in this area are all selected into the preliminary information database, and the corresponding abnormality is marked / Normal mark. Three-phase abnormality at the same time is regarded as a power failure of the substation. The daily abc three-phase 96 hour voltage information is converted into blackout information as the basic information for distribution network monitoring.

\subsection{Calculate the Power Supply of The Line Using the Changed Data of The Collected Information}

After the collected information is obtained, the voltage data of the station area is filtered, calculated, and combined to obtain the power supply data of the distribution network line, which can be further analyzed for frequent power outages, low voltage, etc., and the faults and planned power outages can be distinguished. The process of data analysis and calculation mainly uses Visual Basic for Applications (VBA) technology, this technology can realize the screen switching and statistics of complex logic through a piece of code.

\subsubsection{Data Collection to Power Outage Information Data Processing}

\begin{tabular}{|c|c|c|}
\hline 数据来焦月份 & 记录数三 & 日均 \\
\hline 201701 & 1169033 & 37711 \\
\hline 201702 & 1335430 & 47694 \\
\hline 201703 & 1293140 & 43105 \\
\hline 201704 & 1322403 & 44080 \\
\hline
\end{tabular}

\section{1-4月日采集记录数}

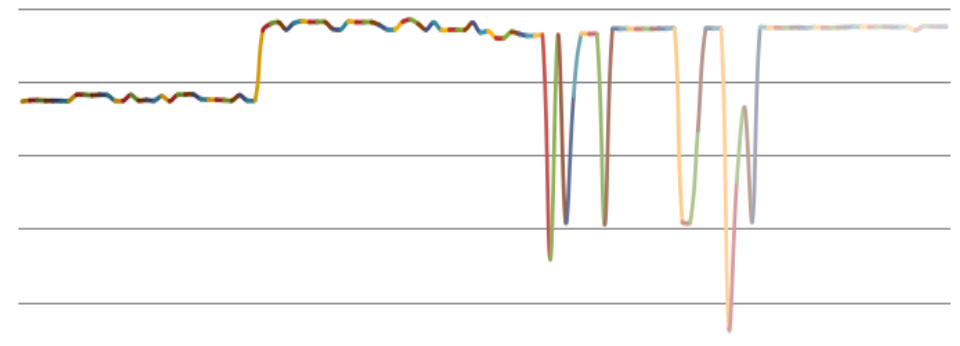

Figure 2 Data collection to data processing of power outage information

Using the VBA technology, the form displayed after processing the information collected by the public transformer and special transformer is as follows:

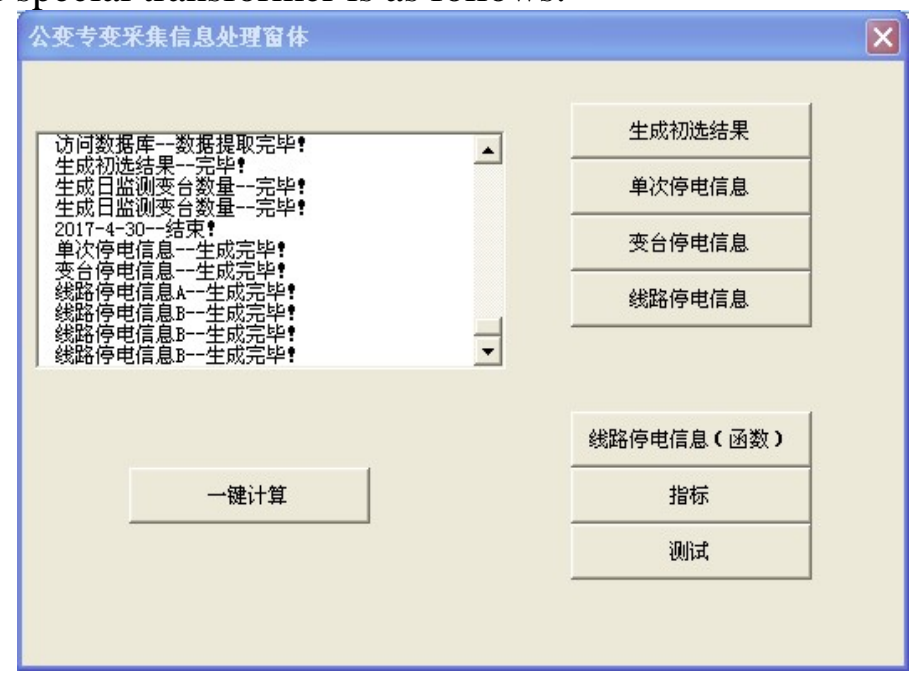

Figure 3 Public information processing form 


\subsubsection{VBA Data Processing}

Through the programming of VBA language, the power outage data of the time zone of the station area is calculated as the power outage time of the station area. The power outage information of the line is calculated through the power outage information of the station area. The summary information of the line outage is as follows:

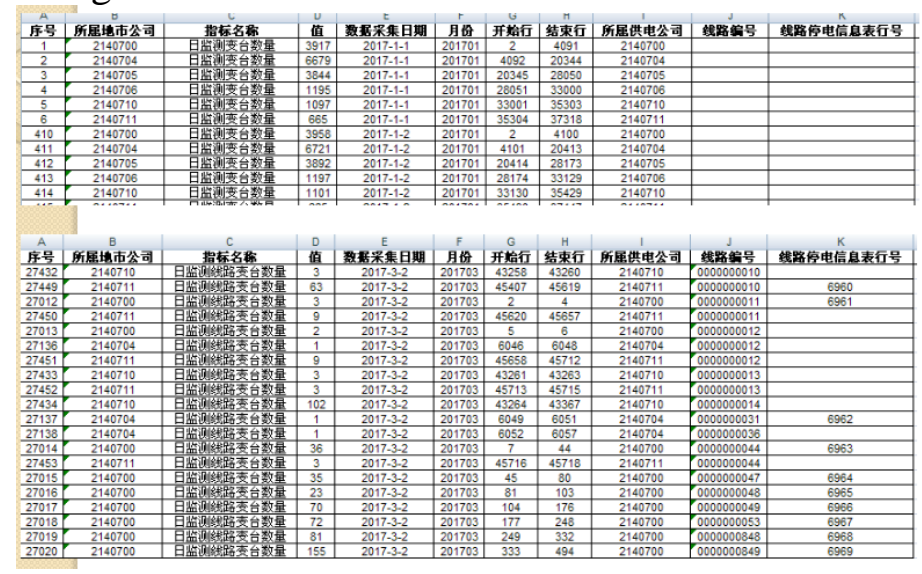

Figure 4 Summary information of line blackout

\section{Practical Application}

The distribution network power supply reliability monitoring and analysis system is used in the monitoring and prediction of frequent power outages in distribution lines in Fuxin area, as follows:

\subsection{Monitoring of The Overall Situation of Frequent Power Outages}

The number of frequent power outage lines is monitored according to the distribution. From January to July 2017, the company has a total of 122 power outage lines, of which 74 lines have repeated power outages more than 2 times, including 58 lines 2-5 times, 6-10 There are 7 sub-lines and 7 over 10 lines. The number of power outages of the 5543 line of the five south line is the highest, reaching 158, and the nature, area, time and cause of power outage of this line need to be further checked.

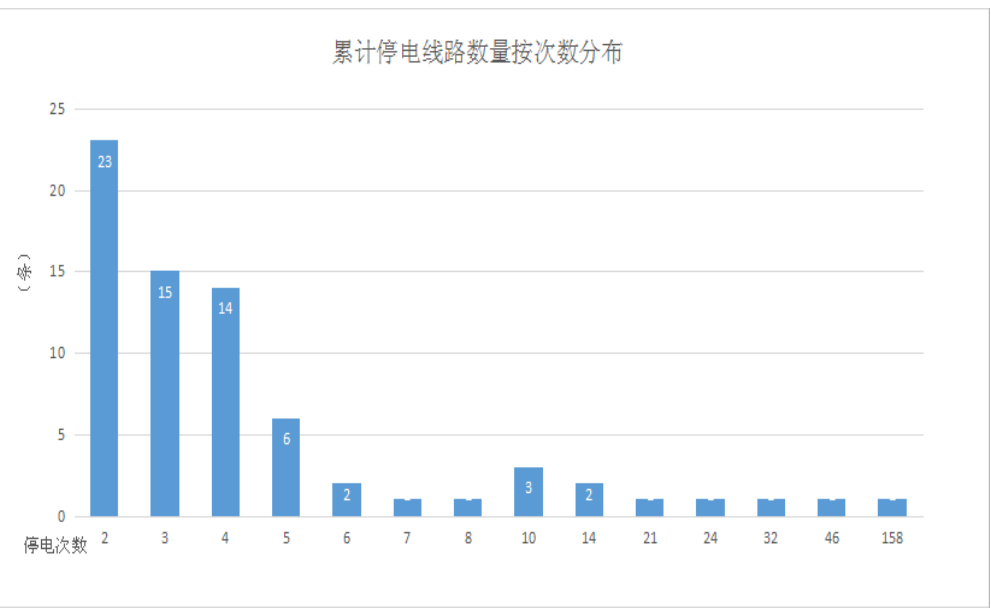

Figure5 Distribution of the company's cumulative number of lines by frequency in 2017

Among them, there are 23 lines with frequent power outages, which are 23, followed by 3 and 4 power outages, with 15 and 14 respectively.

The company has 20 lines with more than 5 power outages. Among them, the number of frequent power outage lines in Haizhou Power Supply Branch is the largest and most obvious in the city. Further verification of the number of power outages of the power outage line learned that Wunan Line 5543 has the most power outages, 159. Can further check the nature of power outage and the cause of power outage based on the monitoring and analysis results. 


\subsection{Monitoring and Analysis of Frequent Power Outages}

From January to July 2017, the lines with frequent power outages of 10 times or more mainly include the following lines:

Table 1 Details of the number of power outages of the company's frequent power outages of 10 or more lines in 2017

\begin{tabular}{|c|c|c|c|c|c|c|c|c|}
\hline Line name & 201701 & 201702 & 201703 & 201704 & 201705 & 201706 & 201707 & Total \\
\hline 10kV Haijiu Line 4743 & 2 & 0 & 2 & 0 & 0 & 3 & 3 & 10 \\
\hline 10kV Unong Line 7046 & 11 & 3 & 7 & 6 & 6 & 7 & 6 & 46 \\
\hline $\begin{array}{c}\text { East Water Source Line } \\
\text { 348 }\end{array}$ & 6 & 0 & 1 & 6 & 2 & 2 & 4 & 21 \\
\hline Fluoride line 042 & 3 & 1 & 1 & 1 & 1 & 2 & 5 & 14 \\
\hline Huashuang & 2 & 0 & 0 & 0 & 2 & 3 & 3 & 10 \\
\hline Jianbei Line 5046 & 4 & 3 & 2 & 0 & 0 & 0 & 5 & 14 \\
\hline Jiandong Line 5042 & 5 & 5 & 1 & 8 & 2 & 3 & 8 & 32 \\
\hline North Line 6444 & 3 & 0 & 1 & 6 & 6 & 3 & 5 & 24 \\
\hline Five South Line 5543 & 23 & 21 & 23 & 23 & 24 & 20 & 24 & 158 \\
\hline Wuxin Line 5541 & 1 & 0 & 1 & 0 & 3 & 4 & 1 & 10 \\
\hline
\end{tabular}

It can be seen from the above table that the Wunan line has the most power outages, reaching 159 times. The four lines of the Wunan line, 10kV Wunong line, Jiandong line, and the fluoride line have power outages every month, of which the first two have even power outages every month. , Indicating that the power supply reliability of some of these two lines has serious problems, and the related conditions of the lines need to be further checked.

The specific time for checking the power outage of the Wunan Line is as follows:

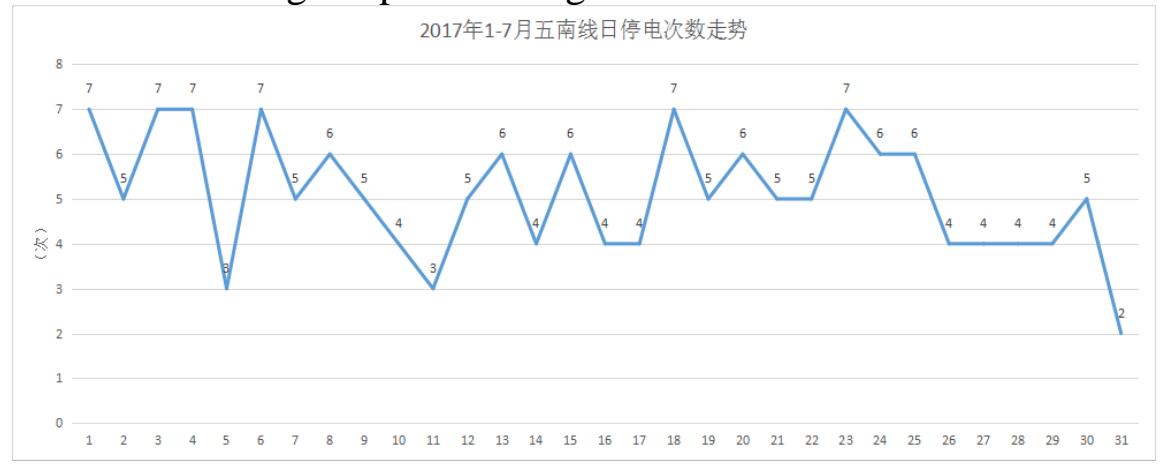

Figure 6 The trend of frequent blackouts on the Wunan Line in 2017

Through the monitoring and analysis of this system, it can be seen that there is a regular power outage on this line, and there are power outages every day. It can be found that there are fault points on this line, and the fault point and fault time are provided to the person in charge of the operation and inspection department. In this way, timely and effective fault handling is carried out to avoid multiple occurrences of frequent power outages.

\section{Conclusion}

The project team did not find any similar platforms by searching on platforms such as HowNet, State Intellectual Property Office, etc. By constructing this system, enterprises can continuously strengthen the effective management of distribution equipment and make the management methods of distribution network more digital and intelligent, effectively reduce the incidence of frequent power outages and improve the reliability of enterprise power supply. The number of power outages in 2019 decreased by $18.2 \%$ compared with the same period in the same period, greatly reducing the economic loss of the enterprise. In addition, with the help of big data analysis methods, Increased the intelligence level of power supply enterprises, making the investment of the enterprise 
more reasonable, increasing the economic benefits of the enterprise, making the distribution network planning more reasonable, the operation more stable, and the maintenance more orderly, thereby winning the trust of customers, reducing the number of customer complaints, and improving quality The quality of service increases the economic benefits of the enterprise, and has wide application prospects and good promotion value.

\section{References}

[1] He Zhengyou, Distribution network analysis and application [m], Beijing: Science Press, 2014, 6 (2): 54-60.

[2] Wei Zheming, Zhang Shuyu, User Power Supply Reliability Management Manual [s], Beijing: China Water Resources and Hydropower Press, 2016, 3 (3): 79 (5): 137.

[3] Wang Jiye, Smart grid big data [m], Beijing: China Electric Power Press, 2017, 2 (2): 80-113, (3): 255-289.

[4] Wang Yimin, User Power Supply Reliability Management Workbook (Second Edition) [s], Beijing: China Electric Power Press, 2009, 4 (2): 34-42, (4): 73-82.

[5] (British) Meyer-Schonberg, (British) Cooke, Big Data Era [m] Zhejiang People 's Publishing House, 2013, (5) 374-383. 